\title{
Interpolation methods of means and orbits
}

\author{
by \\ Mieczyseaw Mastyeo (Poznań)
}

\begin{abstract}
Banach operator ideal properties of the inclusion maps between Banach sequence spaces are used to study interpolation of orbit spaces. Relationships between those spaces and the method-of-means spaces generated by couples of weighted Banach sequence spaces with the weights determined by concave functions and their Janson sequences are shown. As an application we obtain the description of interpolation orbits in couples of weighted $L_{p}$-spaces when they are not described by the $K$-method. We also develop a connection between the method of means with a quasi-parameter and the real method of interpolation generated by the Calderón-Lozanovsky space parameters. Applications to interpolation of operators are also discussed.
\end{abstract}

1. Introduction. The ultimate goal of interpolation theory is the characterization of all relative interpolation spaces with respect to given compatible couples of Banach spaces. A significant role in the study of interpolation spaces is played by interpolation orbits (see [3], [29]). For many specific couples a complete description of interpolation orbits has been achieved through the work of many authors. In almost all known cases there is a simple description in terms of the $K$-functional of the initial couples. Such couples are called relative Calderón couples (or relative Calderón-Mityagin couples) or $K$-monotone couples. Calderón couples therefore play an important role in interpolation theory, since for such couples we may obtain a complete description of all their interpolation spaces (see [3]). Unfortunately the class of Calderón couples is not large. We refer here to the remarkable papers [6], [7] and [18], where Calderón couples of Banach lattices are studied and many references are included.

Although recently great progress has been made in interpolation theory, the problem of describing the interpolation orbits between couples which are not relative Calderón remains a formidable one, even for classical couples of Banach spaces. We refer to [29], where certain positive results in

2000 Mathematics Subject Classification: Primary 46B70.

Key words and phrases: interpolation orbit, method of means, Calderón-Lozanovsky method, weighted Banach lattice, Calderón couple, $K$-method.

The author was supported by KBN Grant 1 P03A 01326. 
this direction are presented. Let us remark that, for couples of $L_{p}$-spaces, the results by Dmitriev [13] seem to have been overlooked. In fact [13] contains a description of the interpolation orbits for certain couples of $L_{p^{-}}$ spaces which are not Calderón couples. More precisely, Dmitriev described interpolation orbits of any element from the couple $\left(L_{p_{0}}\left(u_{0}\right), L_{p_{1}}\left(u_{1}\right)\right)$ into $\left(L_{q_{0}}\left(w_{0}\right), L_{q_{1}}\left(w_{1}\right)\right)$ in the following cases: $1 \leq p_{0}, p_{1} \leq \infty$ and $q_{0}=q_{1}=1$, $1 \leq p_{1}, q_{0} \leq \infty$ and $p_{0}=q_{1}=1$. Further, Ovchinnikov [30] obtained a description of the interpolation orbits for elements with a quasi-power $K$ functional. Recently a complete description of interpolation orbits in couples of $L_{p}$-spaces was presented in the remarkable paper of Ovchinnikov [31].

The aim of this paper is to extend Ovchinnikov's results to the setting of couples of Banach lattices. More generally, the object of this paper is to show how Banach operator ideals can be applied to study interpolation orbits. Following [26], we use the Banach ideal of $(E, 1)$-summing operators (cf. also [11]) to study interpolation of operators acting between abstract real method spaces.

We present general results on the relationship between interpolation methods of orbits and means. When specialized to $L_{p}$-spaces, our results extend and simplify those of Ovchinnikov [31]. The main results of this paper were circulated in the preprint [25].

Before sketching the content of the paper in more detail it will be useful to establish some basic notation and definitions. For unexplained notions from interpolation theory we refer to [1], [3]. Throughout the paper let $(\Omega, \Sigma, \mu)$ be a complete $\sigma$-finite measure space. Let $L_{0}(\mu)$ denote, as usual, the space of equivalence classes of real-valued measurable functions on $\Omega$, equipped with the topology of convergence in $\mu$ measure on sets of finite measure. By a Banach lattice on $\Omega$ we mean a Banach space $X$ which is a subspace of $L_{0}(\mu)$ such that there exists $u \in X$ with $u>0$ and if $|f| \leq|g|$ $\mu$-a.e., where $g \in X$ and $f \in L_{0}(\mu)$, then $f \in X$ and $\|f\|_{X} \leq\|g\|_{X}$.

In the special case when $\Omega=\mathbb{J}$ and $\mu$ is the counting measure, where $\mathbb{J}=-\mathbb{N} \cup\{0\}$ or $\mathbb{J}=\mathbb{Z}$ or $\mathbb{J}=\mathbb{N} \cup\{0\}$, a Banach lattice on $\Omega$ is called a Banach sequence space on $\mathbb{J}$.

If $X$ is a Banach lattice on $(\Omega, \mu)$ and $w \in L^{0}(\mu)$ with $w>0 \mu$-a.e., we define the weighted Banach lattice $X(w)$ by setting

$$
\|x\|_{X(w)}:=\|x w\|_{X} .
$$

If $\bar{X}=\left(X_{0}, X_{1}\right)$ and $\bar{Y}=\left(Y_{0}, Y_{1}\right)$ are couples of Banach spaces, we let $L(\bar{X}, \bar{Y})$ be the Banach space of all linear operators $T: \bar{X} \rightarrow \bar{Y}$ (which means, as usual, that $T: X_{0}+X_{1} \rightarrow Y_{0}+Y_{1}$ is linear and the restrictions $\left.T\right|_{X_{j}}$ are bounded mappings from $X_{j}$ to $Y_{j}$ for $\left.j=0,1\right)$ equipped with the norm

$$
\|T\|_{\bar{X} \rightarrow \bar{Y}}:=\max \left\{\|T\|_{X_{0} \rightarrow Y_{0}},\|T\|_{X_{1} \rightarrow Y_{1}}\right\} .
$$


The elements $x \in X_{0}+X_{1}$ and $y \in Y_{0}+Y_{1}$ are said to be orbitally equivalent with respect to the couples $\bar{X}$ and $\bar{Y}$ if there exist linear operators $T: \bar{X} \rightarrow \bar{Y}$ and $S: \bar{Y} \rightarrow \bar{X}$ such that $T x=y, S y=x$.

If $\bar{A}$ is a Banach couple and $0 \neq a \in A_{0}+A_{1}$, then the interpolation orbit $\operatorname{Orb}(a, \bar{A} \rightarrow \bar{X})$ of the element $a$ in the couple $\bar{X}$ is a Banach space consisting of all elements of the form $x=T a$ for some $T: \bar{A} \rightarrow \bar{X}$, equipped with the norm

$$
\|x\|=\inf \left\{\|T\|_{\bar{A} \rightarrow \bar{X}} ; x=T a\right\} .
$$

The $K$-functional is defined on $X_{0}+X_{1}$ by

$$
K(s, t, x ; \bar{X}):=\inf \left\{s\left\|x_{0}\right\|_{X_{0}}+t\left\|x_{1}\right\|_{X_{1}} ; x=x_{0}+x_{1}\right\}, \quad s, t>0 .
$$

In what follows, we write for short $K(t, x ; \bar{X})$ instead of $K(1, t, x ; \bar{X})$. The Banach spaces $X$ and $Y$, intermediate with respect to $\bar{X}$ and $\bar{Y}$ respectively, are said to be relative $K$-monotone whenever $x \in X$ and $y \in Y_{0}+Y_{1}$ with $K(t, y ; \bar{Y}) \leq K(t, x ; \bar{X})$ for all $t>0$ imply that $y \in Y$. If all relative interpolation spaces with respect to $\bar{X}$ and $\bar{Y}$ are relative $K$-monotone, then we say that interpolation is described by the $K$-method (or equivalently that $\bar{X}$ and $\bar{Y}$ are relative Calderón couples). If this property holds for $\bar{X}=\bar{Y}$, then $\bar{X}$ is said to be a Calderón couple. Clearly, $\bar{X}$ and $\bar{Y}$ are relative Calderón couples provided for any $x \in X_{0}+X_{1}$ and $y \in Y_{0}+Y_{1}$ satisfying $K(t, y ; \bar{Y}) \leq K(t, x ; \bar{X})$ for all $t>0$ there exists an operator $T: \bar{X} \rightarrow \bar{Y}$ such that $\bar{T} x=y$. If there exists a constant $\lambda$, independent of $x$ and $y$, such that $\|T\|_{\bar{X} \rightarrow \bar{Y}} \leq \lambda$, we say that $\bar{X}$ and $\bar{Y}$ are relative uniform Calderón couples.

It is well known that if $\bar{X}$ and $\bar{Y}$ are relative Calderón couples, then $\bar{Y}$ is relatively complete, i.e., the Gagliardo (relative) completion $\bar{Y}^{c}:=\left(Y_{0}^{c}, Y_{1}^{c}\right)$ coincides with $\bar{Y}$ (see, e.g., [3], [8], [6]). Recall that if $X$ is an intermediate Banach space with respect to a couple $\bar{X}$, then its Gagliardo completion $X^{c}$ is the Banach space of all limits in $X_{0}+X_{1}$ of sequences that are bounded in $X$.

Let us outline briefly the content of the paper. In Section 2, we study the method of means determined by weighted Banach sequence spaces with weights generated by concave functions and their Janson sequences. An equivalent description in terms of the $K$-functional for these spaces is presented. Using a Carlson type inequality, we prove continuous inclusions between spaces generated by the method of means and the Calderón-Lozanovsky method applied to a certain class of couples of Banach lattices satisfying upper or lower lattice estimates. Some applications are shown for weighted Orlicz spaces. In particular, we show that the method of means generated by a concave function $\varphi$ and corresponding weighted $\ell_{p_{0}}$ and $\ell_{p_{1}}$-spaces applied to any couple of weighted Banach lattices $\left(L_{p_{0}}\left(w_{0}\right), L_{p_{1}}\left(w_{1}\right)\right)$ spaces coincides, up to equivalence of norms, with the Calderón-Lozanovsky space $\varphi\left(L_{p_{0}}\left(w_{0}\right), L_{p_{1}}\left(w_{1}\right)\right)$. 
In Section 3, we show applications of Banach operator ideals introduced first in [26] to study interpolation of orbit spaces. Continuous inclusions are shown between these spaces and corresponding spaces generated by the method of means. As a consequence of these results, we provide new couples of Banach lattices which are not relative Calderón couples. Further, we obtain the result of Ovchinnikov [31] on the description of interpolation orbits in couples of weighted $L_{p}$-spaces when they are not described by the $K$-method. We also discuss applications to interpolation of operators.

2. The methods of means and of Calderón-Lozanovsky. In this section we shall deal with vector-valued Banach sequence spaces. Let $E$ be a Banach sequence lattice on $\mathbb{J}$ and let $X$ be a Banach space. The vector sequence $x=\left\{x_{n}\right\}_{n \in \mathbb{J}}$ in $X$ is called strongly E-summable if the corresponding scalar sequence $\left\{\left\|x_{n}\right\|_{X}\right\}$ is in $E$. We denote by $E(X)$ the set of all such sequences in $X$. It forms a Banach space under pointwise operations, and a natural quasi-norm given by

$$
\|x\|_{E(X)}=\left\|\left\{\left\|x_{n}\right\|_{X}\right\}\right\|_{E} .
$$

Throughout the paper a pair $\bar{\Phi}=\left(\Phi_{0}, \Phi_{1}\right)$ of Banach sequence lattices on $\mathbb{J}$ is called a parameter of the method of means if $\Phi_{0} \cap \Phi_{1} \subset \ell_{1}$. The space $J_{\bar{\Phi}}(\bar{X})=J_{\Phi_{0}, \Phi_{1}}(\bar{X})$ built by the method of means consists of all $x \in X_{0}+X_{1}$ which may be represented in the form

$$
\left.x=\sum_{n} u_{n} \quad \text { (convergence in } X_{0}+X_{1}\right)
$$

with $\left\{u_{n}\right\} \in \Phi_{0}\left(X_{0}\right) \cap \Phi_{1}\left(X_{1}\right)$. It is well known that $J_{\bar{\Phi}}(\bar{X})$ is a Banach space under the norm

$$
\|x\|_{J_{\bar{\Phi}}(\bar{X})}=\inf \max \left\{\left\|\left\{u_{n}\right\}\right\|_{\Phi_{0}\left(X_{0}\right)},\left\|\left\{u_{n}\right\}\right\|_{\Phi_{1}\left(X_{1}\right)}\right\}
$$

where the infimum is taken over all representations of $x$ as above (see, e.g., $[3],[19])$.

In this paper we are interested in the special method of means generated by weighted Banach sequence spaces determined by quasi-concave functions. More precisely, let $\varphi \in \mathcal{P}$ (i.e., $\varphi:[0, \infty) \times[0, \infty) \rightarrow[0, \infty), t \mapsto \varphi(1, t)$ is a positive quasi-concave function on $[0, \infty)$ with $\varphi(0,0)=0$ and $\varphi$ is positively homogeneous). Assume that $\varphi \in \mathcal{P}_{0}$ (i.e., $\varphi(1, t) \rightarrow 0$ as $t \rightarrow 0$ and $\varphi(t, 1) \rightarrow 0$ as $t \rightarrow 0)$. A sequence $\left\{t_{n}\right\}_{n \in \mathbb{J}}$ is called a fundamental sequence for $\varphi$ if the following equivalences hold for $\varrho(t):=\varphi(1, t)$ for $t>0$ :

$$
\varrho(t) \asymp \sum_{n} \varrho\left(t_{n}\right) \min \left\{1, t / t_{n}\right\} \asymp \sup _{n} \varrho\left(t_{n}\right) \min \left\{1, t / t_{n}\right\},
$$

or equivalently in terms of $K$-functionals,

$$
\varrho(t) \asymp K\left(t,\left\{\varrho\left(t_{n}\right)\right\} ; \ell_{1}, \ell_{1}\left(1 / t_{n}\right)\right) \asymp K\left(t,\left\{\varrho\left(t_{n}\right)\right\} ; \ell_{\infty}, \ell_{\infty}\left(1 / t_{n}\right)\right) .
$$


Here and throughout the paper, for positive functions $f$ and $g$, we write $f \asymp g$ whenever $f \prec g$ and $g \prec f$, where $f \prec g$ means that there is a constant $c>0$ such that $f \leq c g$.

The construction of fundamental sequences for $\varphi \in \mathcal{P}_{0}$ may be realized as follows (see [17]). For a given $q>1$, we define inductively a sequence $\left\{t_{n}\right\}_{n \in \mathbb{J}}$ with $t_{0}=1$ (which is called the Janson sequence for $\varphi($ or $\varrho$ )) by

$$
\min \left\{\frac{\varrho\left(t_{n+1}\right)}{\varrho\left(t_{n}\right)}, \frac{t_{n+1} \varrho\left(t_{n}\right)}{t_{n} \varrho\left(t_{n+1}\right)}\right\}=q .
$$

Note that for a given $\varphi \in \mathcal{P}_{0}$ Brudnyı and Krugljak used in [3] a little different construction of a sequence $\left\{t_{n}\right\}$ with $t_{0}=1$ (called the Brudny $\breve{\imath}^{-}$ Krugljak sequence for $\varphi$ (or $\varrho))$ :

$$
\frac{\varrho\left(t_{2 n}\right)}{t_{2 n}}=q \frac{\varrho\left(t_{2 n+1}\right)}{t_{2 n+1}} \quad \text { and } \quad \varrho\left(t_{2 n}\right)=q \varrho\left(t_{2 n-1}\right)
$$

with $\varrho(t)=\varphi(1, t)$ for $t>0$ and proved that the sequence $\left\{t_{2 n+1}\right\}$ is a fundamental sequence for $\varphi$.

Assume that $\left\{t_{n}\right\}_{n \in \mathbb{J}}$ is a fundamental sequence for $\varphi \in \mathcal{P}_{0}$. From the equivalences mentioned above, we infer in particular that $\left\{\varrho\left(t_{n}\right)\right\} \in \ell_{1}+$ $\ell_{1}\left(1 / t_{n}\right)$, where $\varrho=\varphi(1, \cdot)$. This implies that for any couple $\left(E_{0}, E_{1}\right)$ of Banach sequence spaces on $\mathbb{J}$ such that $E_{j} \hookrightarrow \ell_{\infty}$ for $j=0,1$ we get

$$
\ell_{\infty} \hookrightarrow E_{0}^{\prime}\left(\varrho\left(t_{n}\right)\right)+E_{1}^{\prime}\left(\varrho\left(t_{n}\right) / t_{n}\right) .
$$

It follows by Köthe duality that $\left(\Phi_{0}, \Phi_{1}\right)=\left(E_{0}\left(1 / \varrho\left(t_{n}\right), E_{1}\left(t_{n} / \varrho\left(t_{n}\right)\right)\right.\right.$ is a parameter of the method of means. In this case the space $J_{\bar{\Phi}}(\bar{X})$ is denoted by $\varphi(\bar{X})_{E_{0}, E_{1}}$. If $E_{0}=\ell_{p_{0}}$ and $E_{1}=\ell_{p_{1}}$ with $1 \leq p_{0}, p_{1} \leq \infty$, we write for

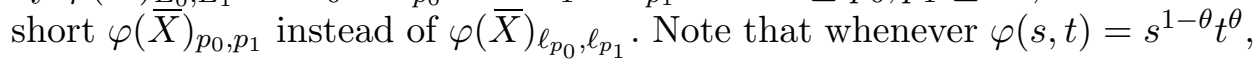
$0<\theta<1$ and $\left\{t_{n}\right\}=\left\{2^{n}\right\}$, then $\varphi(\bar{X})_{p_{0}, p_{1}}$ is the classical Lions-Peetre method-of-means space $J_{\theta, p_{0}, p_{1}}(\bar{X})$ (see [22]). If $E$ is a Banach sequence lattice on $\mathbb{Z}$ and $E_{0}=E_{1}=E$, then $J_{\Phi_{0}, \Phi_{1}}(\bar{X})$ is the classical abstract $J$-space, which is denoted by $J_{E}(\bar{X})$ (see [3], [8], [19]).

The following proposition is an abstract variant of a result presented without proof in [13]. Since the result is not widely known, it seems worthwhile to set it out in detail here.

Proposition 2.1. Let $\left\{t_{n}\right\}_{n \in \mathbb{J}}$ be a fundamental sequence for $\varphi \in \mathcal{P}_{0}$. Assume that $\bar{X}=\left(X_{0}, X_{1}\right)$ is a Banach couple and $\bar{E}=\left(E_{0}, E_{1}\right)$ is a couple of Banach sequence spaces on $\mathbb{J}$ with $E_{j} \hookrightarrow \ell_{\infty}$ for $j=0,1$. Consider the following conditions:

(i) $x \in \varphi\left(X_{0}, X_{1}\right)_{E_{0}, E_{1}}$.

(ii) There exist positive elements $u \in E_{0}$ and $v \in E_{1}\left(t_{n}\right)$ such that

$$
K(t, x ; \bar{X}) \leq K\left(t, a_{\varphi} ; \ell_{1}(u), \ell_{1}(v)\right)
$$

for all $t>0$, where $a_{\varphi}=\left\{\varphi\left(1, t_{n}\right)\right\}$. 
Then (i) implies (ii), and (ii) implies (i) provided that $\bar{X}$ is relatively complete.

Proof. (i) $\Rightarrow\left(\right.$ ii): Assume that $x \in \varphi(\bar{X})_{E_{0}, E_{1}}$ and let $\varrho(t)=\varphi(1, t)$ for $t>0$. Then we have

$$
\left.x=\sum_{n} x_{n} \quad \text { (convergence in } X_{0}+X_{1}\right)
$$

with $\left\{x_{n} / \varrho\left(t_{n}\right)\right\} \in E_{0}\left(X_{0}\right)$ and $\left\{t_{n} x_{n} / \varrho\left(t_{n}\right)\right\} \in E_{1}\left(X_{1}\right)$.

Without loss of generality we may assume that $x_{n} \neq 0$ for all $n \in \mathbb{J}$. We define sequences $u=\left\{u_{n}\right\}$ and $v=\left\{v_{n}\right\}$ by setting $u_{n}=\left\{\left\|x_{n}\right\|_{X_{0}} / \varrho\left(t_{n}\right)\right\}$ and $v_{n}=\left\{\left\|x_{n}\right\|_{X_{1}} / \varrho\left(t_{n}\right)\right\}$ for $n \in \mathbb{J}$. Then $u \in E_{0}$ and $v \in E_{1}\left(t_{n}\right)$. Since $E_{0} \hookrightarrow \ell_{\infty}, E_{1} \hookrightarrow \ell_{\infty}$ and $a_{\varphi} \in \ell_{1}+\ell_{1}\left(1 / t_{n}\right)$, it follows that

$$
\sum_{n} \min \left\{u_{n}, v_{n}\right\} \varrho\left(t_{n}\right)<\infty \text {. }
$$

Further, we have

$$
\begin{aligned}
K(t, x ; \bar{X}) & \leq \sum_{n} K\left(t, x_{n} ; \bar{X}\right) \leq \sum_{n} \min \left\{\left\|x_{n}\right\|_{X_{0}}, t\left\|x_{n}\right\|_{X_{1}}\right\} \\
& =\sum_{n} \min \left\{\frac{\left\|x_{n}\right\|_{X_{0}}}{\varrho\left(t_{n}\right)}, \frac{t\left\|x_{n}\right\|_{X_{1}}}{\varrho\left(t_{n}\right)}\right\} \varrho\left(t_{n}\right)=K\left(t, a_{\varphi} ; \ell_{1}(u), \ell_{1}(v)\right) .
\end{aligned}
$$

(ii) $\Rightarrow$ (i): Assume that $\bar{X}=\left(X_{0}, X_{1}\right)$ is a relatively complete couple and that $x \in X_{0}+X_{1}$ satisfies

$$
K(t, x ; \bar{X}) \leq K\left(t, a_{\varphi} ; \ell_{1}(u), \ell_{1}(v)\right)
$$

for any $t>0$ and positive elements $u=\left\{u_{n}\right\} \in E_{0}$ and $v=\left\{v_{n}\right\} \in E_{1}\left(t_{n}\right)$. Since $\left(\ell_{1}(u), \ell_{1}(v)\right)$ and $\left(X_{0}, X_{1}\right)$ are relative uniform Calderón couples (see, e.g., [3], [8]), there exists an operator $T:\left(\ell_{1}(u), \ell_{1}(v)\right) \rightarrow\left(X_{0}, X_{1}\right)$ such that $\|T\| \leq \gamma$ and $x=T a_{\varphi}$, where $\gamma=\gamma(\bar{X})$ is the $K$-divisibility constant for $\bar{X}$. Clearly,

$$
\left.x=\sum_{n} \varrho\left(t_{n}\right) T e_{n} \quad \text { (convergence in } X_{0}+X_{1}\right) .
$$

Put $x_{n}=\varrho\left(t_{n}\right) T e_{n}$ for $n \in \mathbb{J}$. Then we have

$$
\frac{\left\|x_{n}\right\|_{X_{0}}}{\varrho\left(t_{n}\right)}=\left\|T e_{n}\right\|_{X_{0}} \leq \gamma u_{n}
$$

and

$$
\frac{t_{n}\left\|x_{n}\right\|_{X_{1}}}{\varrho\left(t_{n}\right)}=t_{n}\left\|T e_{n}\right\|_{X_{1}} \leq \gamma t_{n} v_{n}
$$

Since $\left\{u_{n}\right\} \in E_{0}$ and $\left\{v_{n}\right\} \in E_{1}\left(t_{n}\right)$, we have $x \in \varphi\left(X_{0}, X_{1}\right)_{E_{0}, E_{1}}$, and so the proof is complete.

Corollary 2.2. Let $\left\{t_{n}\right\}_{n \in \mathbb{J}}$ be the Janson sequence for $\varphi \in \mathcal{P}_{0}$. If $\bar{X}=\left(X_{0}, X_{1}\right)$ is a relatively complete Banach couple, then $\varphi\left(X_{0}, X_{1}\right)_{E_{0}, E_{1}}$ 
consists of all $x \in X_{0}+X_{1}$ such that

$$
\left\{K\left(s_{n}, x ; \bar{X}\right)\right\} \in \varphi\left(G_{0}, G_{1}\left(1 / s_{n}\right)\right)_{E_{0}, E_{1}}
$$

for a certain couple $\left(G_{0}, G_{1}\right)$ of Banach sequence spaces on $\mathbb{J}$ with $\ell_{1} \hookrightarrow$ $G_{j} \hookrightarrow \ell_{\infty}(j=0,1)$, where $\left\{s_{n}\right\}$ is the Janson sequence for $K(\cdot, x ; \bar{X})$.

Proof. First let us note that if $\left\{s_{n}\right\}_{n \in \mathbb{J}}$ is the Janson sequence for $\psi:=$ $K(\cdot, x ; \bar{X})$, then for any couple $\left(G_{0}, G_{1}\right)$ of Banach sequence spaces on $\mathbb{J}$ such that $\ell_{1} \hookrightarrow G_{j} \hookrightarrow \ell_{\infty}(j=0,1)$, we have

$$
K\left(t,\left\{\psi\left(s_{n}\right)\right\} ; G_{0}, G_{1}\left(1 / s_{n}\right)\right) \asymp \psi(t)
$$

by known equivalences (see, e.g., [29])

$$
K\left(t,\left\{\psi\left(s_{n}\right)\right\} ; \ell_{1}, \ell_{1}\left(1 / s_{n}\right)\right) \asymp K\left(t,\left\{\psi\left(s_{n}\right)\right\} ; \ell_{\infty}, \ell_{\infty}\left(1 / s_{n}\right)\right) \asymp \psi(t) .
$$

Now assume that $x \in \varphi\left(X_{0}, X_{1}\right)_{E_{0}, E_{1}}$. Then by Proposition 2.1, there exist positive elements $u \in E_{0}$ and $v \in E_{1}\left(t_{n}\right)$ such that

$$
K(t, x ; \bar{X}) \leq K\left(t, a_{\varphi} ; \ell_{1}(u), \ell_{1}(v)\right)
$$

where $a_{\varphi}=\left\{\varphi\left(1, t_{n}\right)\right\}$. Combining the above relations, we find that

$$
K\left(t,\left\{\psi\left(s_{n}\right)\right\} ; G_{0}, G_{1}\left(1 / s_{n}\right)\right) \prec K\left(t, a_{\varphi} ; \ell_{1}(u), \ell_{1}(v)\right) .
$$

Now applying again Proposition 2.1, we obtain

$$
\left\{K\left(s_{n}, x ; \bar{X}\right)\right\} \in \varphi\left(G_{0}, G_{1}\left(1 / s_{n}\right)\right)_{E_{0}, E_{1}} .
$$

If we assume that the above relation holds for a certain couple $\left(G_{0}, G_{1}\right)$ of Banach sequence spaces on $\mathbb{J}$ with $\ell_{1} \hookrightarrow G_{j} \hookrightarrow \ell_{\infty}(j=0,1)$, then by Proposition 2.1, we conclude that the inequality

$$
K\left(t,\left\{\psi\left(s_{n}\right)\right\} ; G_{0}^{c}, G_{1}\left(1 / s_{n}\right)^{c}\right) \leq K\left(t, a_{\varphi} ; \ell_{1}(u), \ell_{1}(v)\right)
$$

holds for some $u \in E_{0}$ and $v \in E_{1}\left(t_{n}\right)$. This implies that

$$
K(t, x ; \bar{X}) \prec K\left(t, a_{\varphi} ; \ell_{1}(u), \ell_{1}(v)\right),
$$

and in consequence $x \in \varphi\left(X_{0}, X_{1}\right)_{E_{0}, E_{1}}$, by Proposition 2.1 .

Further on we shall deal with the Calderón-Lozanovsky spaces. We show that under certain geometrical conditions, continuous inclusions hold between the method-of-means spaces and the Calderón-Lozanovsky spaces. In the case of Banach couples of $L_{p}$-spaces, we get precise equalities between these spaces. Certain results in this direction were shown in [26].

We recall first that if $\bar{X}=\left(X_{0}, X_{1}\right)$ is a couple of Banach lattices on $(\Omega, \mu)$ and $\psi \in \mathcal{P}$ is a concave function then the Calderón-Lozanovsky space $\psi(\bar{X})=\psi\left(X_{0}, X_{1}\right)$ consists of all $x \in L^{0}(\mu)$ such that $|x|=\psi\left(\left|x_{0}\right|,\left|x_{1}\right|\right)$ for some $x_{j} \in X_{j}, j=0,1$. The space $\psi(\bar{X})$ is a Banach lattice equipped with the norm (see [23])

$$
\|x\|=\inf \left\{\max \left\{\left\|x_{0}\right\|_{X_{0}},\left\|x_{1}\right\|_{X_{1}}\right\}:|x|=\psi\left(\left|x_{0}\right|,\left|x_{1}\right|\right), x_{0} \in X_{0}, x_{1} \in X_{1}\right\} .
$$

In what follows we need a variant of Carlson's inequality (cf. [16], [20]). 
Proposition 2.3. Assume that $q>1, \varphi \in \mathcal{P}_{0}$ and $\left\{t_{n}\right\}_{n \in \mathbb{J}}$ is the Janson (respectively, the Brudny̆-Krugljak) sequence for $\varphi$. Then, for any finite positive sequence $\left\{u_{n}\right\} \in \ell_{1}(\mathbb{J})$, the following inequality holds with $\varrho(t)=$ $\varphi(1, t)$ for $t>0$ :

$$
\sum_{n} u_{n} \leq \frac{q-1}{q+1} \varphi\left(\sup _{n} \frac{u_{n}}{\varrho\left(t_{n}\right)}, \sup _{n} \frac{t_{n} u_{n}}{\varrho\left(t_{n}\right)}\right)
$$

and respectively:

$$
\sum_{n} u_{n} \leq \frac{q-1}{q+1} \varphi\left(\sup _{n} \frac{u_{n}}{\varrho\left(t_{2 n+1}\right)}, \sup _{n} \frac{t_{2 n+1} u_{n}}{\varrho\left(t_{2 n+1}\right)}\right) .
$$

Proof. Assume that $\left\{t_{n}\right\}$ is the Janson sequence for $\varphi$. Put

$$
A:=\sup _{n} \frac{u_{n}}{\varrho\left(t_{n}\right)}, \quad B:=\sup _{n} \frac{t_{n} u_{n}}{\varrho\left(t_{n}\right)} .
$$

There exists $k \in \mathbb{J}$ such that $A / B \in\left[t_{k}, t_{k+1}\right)$. Since $t \mapsto \varrho(t)$ is a nondecreasing function and $\varphi\left(1, t_{n}\right) \leq q^{-1} \varphi\left(1, t_{n+1}\right)$, we obtain

$$
\begin{aligned}
\sum_{n \leq k} u_{n} & \leq A \sum_{n \leq k} \varphi\left(1, t_{n}\right) \leq A\left(1+q^{-1}+q^{-2}+\cdots\right) \varphi\left(1, t_{k}\right) \\
& \leq \frac{q}{q-1} A \varphi(1, B / A)=\frac{q}{q-1} \varphi(A, B) .
\end{aligned}
$$

Similarly, by the fact that $t \mapsto \varphi\left(t^{-1}, 1\right)$ is non-increasing and

$$
\frac{\varphi\left(1, t_{n+1}\right)}{t_{n+1}} \leq \frac{1}{q} \frac{\varphi\left(1, t_{n}\right)}{t_{n}}
$$

we get

$$
\begin{aligned}
\sum_{n>k} u_{n} & \leq B \sum_{n>k} \frac{\varrho\left(t_{n}\right)}{t_{n}} \leq B\left(q^{-1}+q^{-2}+\cdots\right) \frac{\varphi\left(1, t_{k}\right)}{t_{k}} \\
& \leq \frac{B}{q-1} \varphi\left(t_{k}^{-1}, 1\right) \leq \frac{B}{q-1} \varphi(A / B, 1)=\frac{1}{q-1} \varphi(A, B) .
\end{aligned}
$$

Since $\sum_{n} u_{n}=\sum_{n \leq k} u_{n}+\sum_{n>k} u_{n}$, the above estimates yield the desired inequality. If $\left\{t_{n}\right\}$ is the Brudny - Krugljak sequence the proof is similar.

In order to present the next results we need a generalization of the well known notions of upper and lower $p$-estimates (cf. [21, pp. 82-84]). Let $E$ be a Banach sequence space on $\mathbb{J}$. A Banach lattice $X$ is said to satisfy an upper (resp., a lower) E-estimate if the following continuous inclusions between vector-valued Banach spaces hold:

$$
E(X) \hookrightarrow X\left[\ell_{\infty}\right]
$$

respectively

$$
X\left[\ell_{1}\right] \hookrightarrow E(X)
$$


The constants of the above embeddings are called the upper (resp., lower) E-estimate constants and are denoted by $u_{E}$ (resp., $l_{E}$ ).

Recall that $\varphi \in \mathcal{P}$ is called non-degenerate if the ranges of the functions $\varphi(\cdot, 1)$ and $\varphi(1, \cdot)$ coincide with $(0, \infty)$.

THeOREM 2.4. Let $\left(E_{0}, E_{1}\right)$ be Banach sequence spaces such that $\ell_{1} \hookrightarrow$ $E_{j} \hookrightarrow \ell_{\infty}$ for $j=0,1$. Let $X_{0}$ and $X_{1}$ be Banach lattices on a measure space $(\Omega, \mu)$. Then the following continuous inclusions hold with constants depending on the corresponding $E_{j}$-estimates:

(i) If $X_{j}$ satisfies an upper $E_{j}$-estimate $(j=0,1)$ and $\left\{t_{n}\right\}$ is the Janson or Brudny̆̌-Krugljak sequence for $\varphi \in \mathcal{P}_{0}$, then

$$
\varphi\left(X_{0}, X_{1}\right)_{E_{0}, E_{1}} \hookrightarrow \varphi\left(X_{0}, X_{1}\right) .
$$

(ii) If $X_{j}$ satisfies a lower $E_{j}$-estimate $(j=0,1)$ and $\left\{t_{n}\right\}$ is the Brudny̆Krugljak sequence for non-degenerate $\varphi \in \mathcal{P}$, then

$$
\varphi\left(X_{0}, X_{1}\right) \hookrightarrow \varphi\left(X_{0}, X_{1}\right)_{E_{0}, E_{1}} .
$$

Proof. (i) Fix $q>1$ and assume without loss of generality that $\left\{t_{n}\right\}_{n \in \mathbb{J}}$ is the Janson sequence for $\varrho=\varphi(1, \cdot)$. Let $x \in \varphi\left(X_{0}, X_{1}\right)_{E_{0}, E_{1}}$ with $\|x\|<1$. Then

$$
\left.x=\sum_{n} u_{n} \quad \text { (convergence in } X_{0}+X_{1}\right)
$$

with $\left\|\left\{u_{n} / \varrho\left(t_{n}\right)\right\}\right\|_{E_{0}\left(X_{0}\right)} \leq 1$ and $\left\|\left\{t_{n} u_{n} / \varrho\left(t_{n}\right)\right\}\right\|_{E_{1}\left(X_{1}\right)} \leq 1$. This implies that the above series is absolutely convergent in $X_{0}+X_{1}$. Thus, in particular, $\left\{\left|u_{n}(\omega)\right|\right\} \in \ell_{1}$ for almost all $\omega \in \Omega$. Since $X_{j}$ satisfies an upper $E_{j}$-estimate $(j=0,1)$, we get

$$
x_{0}(\cdot):=\sup _{n} \frac{\left|u_{n}(\cdot)\right|}{\varrho\left(t_{n}\right)} \in X_{0}, \quad x_{1}(\cdot):=\sup _{n} \frac{t_{n}\left|u_{n}(\cdot)\right|}{\varrho\left(t_{n}\right)} \in X_{1},
$$

with $\left\|x_{j}\right\|_{X_{j}} \leq u_{E_{j}}$, where $u_{E_{j}}$ is the constant of the embedding $E_{j}\left(X_{j}\right) \hookrightarrow$ $X_{j}\left[\ell_{\infty}\right], j=0,1$. Combining these facts with the Carlson inequality we obtain

$$
\|x\| \leq \sum_{n}\left|u_{n}\right| \leq C \varphi\left(x_{0}, x_{1}\right)
$$

with $C=(q+1) /(q-1)$. This shows that $x \in \varphi\left(X_{0}, X_{1}\right)$ and $\|x\| \leq$ $C \max \left\{u_{E_{0}}, u_{E_{1}}\right\}$.

(ii) Let $0 \leq x \in \varphi(\bar{X})$ and $\|x\|_{\varphi(\bar{X})}<1$. Then $x=\varphi\left(x_{0}, x_{1}\right)$ for some $0 \leq x_{j} \in X_{j}$ such that $\left\|x_{j}\right\|_{X_{j}}<1, j=0,1$. Since $\varphi \in \mathcal{P}_{0}$, it follows that the support of $x$ is contained in the intersection of the supports of $x_{0}$ and $x_{1}$. Hence, we may assume without loss of generality that $x, x_{0}, x_{1}$ are not equal to zero on the domain $\Omega$. 
Let $\left\{t_{k}\right\}$ be the Brudny̌-Krugljak sequence for $\varphi$. Since $\varphi$ is non-degenerate, $\left\{t_{k}\right\}$ is defined on $\mathbb{Z}$. Define for any $k \in \mathbb{Z}$,

$$
A_{k}=\left\{\omega \in \Omega ; t_{2 k} \leq x_{1}(\omega) / x_{0}(\omega)<t_{2 k+2}\right\}
$$

and

$$
y_{k}=x \chi_{A_{k}}, \quad u_{k}=x_{0} \chi_{A_{k}}, \quad v_{k}=x_{1} \chi_{A_{k}} .
$$

It is easily seen that for any $k \in \mathbb{Z}$, we have

$$
y_{k} \leq q \varrho\left(t_{2 k+1}\right) u_{k}
$$

and

$$
y_{k} \leq \frac{\varrho\left(t_{2 k}\right)}{t_{2 k}} x_{1} \chi_{A_{k}} \leq q \frac{\varrho\left(t_{2 k+1}\right)}{t_{2 k+1}} v_{k} .
$$

This implies that for any integer $n$, we have

$$
0 \leq \sum_{k \leq-n} y_{k} \leq \varrho\left(t_{-2 n+1}\right) \sum_{k \leq-n} \frac{y_{k}}{\varrho\left(t_{2 k+1}\right)} \leq q \varphi\left(1, t_{-2 n+1}\right) x_{0}
$$

and

$$
0 \leq \sum_{k \geq n} y_{k} \leq \frac{\varrho\left(t_{2 n+1}\right)}{t_{2 n+1}} \sum_{k \geq n} \frac{t_{2 k+1} y_{k}}{\varrho\left(t_{2 k+1}\right)} \leq \varphi\left(t_{2 n+1}^{-1}, 1\right) x_{1} .
$$

Combining the above estimates, we get

$$
\begin{aligned}
\left\|x-\sum_{k=-M}^{N} y_{k}\right\|_{X_{0}+X_{1}} & \leq\left\|\sum_{k=-\infty}^{-M-1} y_{k}\right\|_{X_{0}}+\left\|\sum_{k=N+1}^{-\infty} y_{k}\right\|_{X_{1}} \\
& \leq q \varphi\left(1, t_{-2(M+1)+1}\right)+q \varphi\left(t_{2(N+1)}^{-1}, 1\right)
\end{aligned}
$$

for any positive integers $M$ and $N$. Since $\varphi$ is non-degenerate, $t_{-M} \rightarrow 0$ as $M \rightarrow \infty$ and $t_{N} \rightarrow \infty$ as $N \rightarrow \infty$. This implies that the series $\sum_{n} x_{n}$ with $x_{2 n}:=0$ and $x_{2 n+1}:=y_{n}$ converges to $x$ in $X_{0}+X_{1}$. Further, by the fact that $\left\{A_{n}\right\}$ is a sequence of pairwise disjoint measurable subsets whose union is equal to $\Omega$, we have

$$
\sum_{n} \frac{\left|x_{n}\right|}{\varrho\left(t_{n}\right)}=\sum_{n} \frac{y_{n}}{\varrho\left(t_{2 n+1}\right)} \leq \sum_{n} u_{n} \leq q x_{0}
$$

and

$$
\sum_{n} \frac{t_{n}\left|x_{n}\right|}{\varrho\left(t_{n}\right)}=\sum_{n} \frac{t_{2 n+1} y_{n}}{\varrho\left(t_{2 n+1}\right)} \leq q \sum_{n} v_{n} \leq q x_{1} .
$$

Now assume that $X_{j}$ satisfies a lower $E_{j}$-estimate $(j=0,1)$. Combining the above estimates, we obtain

$$
\left\{x_{n} / \varrho\left(t_{n}\right)\right\} \in E_{0} \quad \text { and } \quad\left\{t_{n} x_{n} / \varrho\left(t_{n}\right)\right\} \in E_{1} .
$$

Since $x_{n} \in X_{0} \cap X_{1}$, we get $x \in \varphi\left(X_{0}, X_{1}\right)_{E_{0}, E_{1}}$. 
Corollary 2.5. Let $\left(L_{M_{0}}\left(w_{0}\right), L_{M_{1}}\left(w_{1}\right)\right)$ be a couple of weighted Orlicz spaces and $\varphi \in \mathcal{P}_{0}$. Then the following statements are true:

(i) If $M_{j}$ is submultiplicative for $j=0,1$ (i.e., $M_{j}(s t) \leq C M_{j}(s) M_{j}(t)$ for some $C>0$ and all $s, t>0)$ then

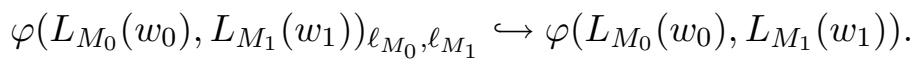

(ii) If $M_{j}$ is supermultiplicative for $j=0,1$ (i.e., $M_{j}(s t) \geq C M_{j}(s) M_{j}(t)$ for some $C>0$ and all $s, t>0)$ then

$$
\varphi\left(L_{M_{0}}\left(w_{0}\right), L_{M_{1}}\left(w_{1}\right)\right) \hookrightarrow \varphi\left(L_{M_{0}}\left(w_{0}\right), L_{M_{1}}\left(w_{1}\right)\right)_{\ell_{M_{0}}, \ell_{M_{1}}} .
$$

Proof. It follows by [11] that if an Orlicz function $M$ is submultiplicative (resp., supermultiplicative), then any weighted Orlicz space $L_{M}(w)$ satisfies an upper (resp., a lower) $\ell_{M}$-estimate. Apply the previous proposition to get what we want.

Since any $L_{p}$-space with $1 \leq p \leq \infty$ satisfies both a lower $p$-estimate and an upper $p$-estimate for any $1 \leq p \leq \infty$, the following corollary is an immediate consequence of Theorem 2.4. We note that this result was also obtained in [31]; however the proof was more complicated. Namely, it used the $K$-divisibility property and the Bennett-Carl result which says that the inclusion map $\ell_{p} \hookrightarrow \ell_{\infty}$ is $(p, 1)$-summing for any $1 \leq p<\infty$ (see [1]).

Corollary 2.6. For any couple $\left(L_{p_{0}}\left(w_{0}\right), L_{p_{1}}\left(w_{1}\right)\right)$ of weighted $L_{p}$ spaces and any $\varphi \in \mathcal{P}_{0}$,

$$
\varphi\left(L_{p_{0}}\left(w_{0}\right), L_{p_{1}}\left(w_{1}\right)\right)_{p_{0}, p_{1}}=\varphi\left(L_{p_{0}}\left(w_{0}\right), L_{p_{1}}\left(w_{1}\right)\right)
$$

with constants of equivalence of norms independent of $\varphi$.

It is well known (see, e.g., [28]) that for any $\varphi \in \mathcal{P}$ and any couple $\left(L_{p_{0}}\left(w_{0}\right), L_{p_{1}}\left(w_{1}\right)\right)$ on $(\Omega, \mu)$ with $1 \leq p_{0}<p_{1} \leq \infty$ the Calderón-Lozanovsky space $\varphi\left(L_{p_{0}}\left(w_{0}\right), L_{p_{1}}\left(w_{1}\right)\right)$ coincides up to equivalence of norms with the generalized Orlicz space of all $f \in L^{0}(\mu)$ such that

$$
\int_{\Omega} M\left(\left(w_{1}^{1 / p_{0}} w_{0}^{-1 / p_{1}}\right)^{q}|f| / \lambda\right)\left(w_{0} / w_{1}\right)^{q} d \mu<\infty
$$

for some $\lambda>0$. Here $1 / q=1 / p_{0}-1 / p_{1}$ and $M$ is an Orlicz function such that $M^{-1}(t) \asymp \varphi\left(t^{1 / p_{0}}, t^{1 / p_{1}}\right)$ for $t>0$.

We conclude this section by showing how Proposition 2.1 and Corollary 2.6 may be applied to prove a variant of the Lions-Peetre formula which describes certain method-of-means spaces as the corresponding $K$-method spaces generated by a quasi-power function parameter. We note that this kind of result has also been proved in a quite different way by Fan [14] for integral variants of the abstract $K$ - and $J$-methods of interpolation. Recall that if $\bar{X}=\left(X_{0}, X_{1}\right)$ is a couple of Banach spaces and $E$ is a Banach sequence space on $\mathbb{Z}$ such that $\left\{\min \left(1,2^{n}\right)\right\} \in E$, then the $K$-method space is 
a Banach space $\bar{X}_{E}$ which consists of all $x \in X_{0}+X_{1}$ such that $\left\{K\left(2^{n}, x ; \bar{X}\right)\right\}$ $\in E$. The space is equipped with the norm $\|x\|=\left\|\left\{K\left(2^{n}, x ; \bar{X}\right)\right\}\right\|_{E}$.

In order to present the final result of this section, we also recall that a function $\varphi \in \mathcal{P}$ is called a quasi-power (written $\varphi \in \mathcal{P}^{+-}$) if the dilatation indices $\delta_{\varrho}$ and $\gamma_{\varrho}$ of the function $\varrho=\varphi(1, \cdot)$ satisfy $0<\delta_{\varrho} \leq \gamma_{\varrho}<1$ (see, e.g., [17], [19]).

Theorem 2.7. Let $\left(X_{0}, X_{1}\right)$ be a Banach couple. Let $\varphi\left(X_{0}, X_{1}\right)_{p_{0}, p_{1}}$ be generated by $\varphi \in \mathcal{P}^{+-}$and the fundamental sequence $\left\{2^{n}\right\}_{n \in \mathbb{Z}}$. Then

$$
\varphi\left(X_{0}, X_{1}\right)_{p_{0}, p_{1}}=\left(X_{0}, X_{1}\right)_{\varphi\left(\ell_{p_{0}}, \ell_{p_{1}}\left(2^{-n}\right)\right)} .
$$

Proof. We show the following claim: $\varphi \in \mathcal{P}^{+-}$implies $\varphi\left(\ell_{p_{0}}, \ell_{p_{1}}\left(2^{-n}\right)\right)$ is a parameter of the real method (for definition see, e.g., [29, p. 432]). In fact, it may be easily shown that $\varphi \in \mathcal{P}^{+-}$implies that

$$
\varphi(s, t) \asymp \psi\left(s^{1-\theta_{0}} t^{\theta_{0}}, s^{1-\theta_{1}} t^{\theta_{1}}\right)
$$

for some $\psi \in \mathcal{P}$ and $0<\theta_{j}<1, j=0,1$. This yields

$$
\varphi\left(\ell_{p_{0}}, \ell_{p_{1}}\left(2^{-n}\right)\right)=\psi\left(\ell_{p_{\theta_{0}}}\left(2^{-n \theta_{0}}\right), \ell_{p_{\theta_{1}}}\left(2^{-n \theta_{1}}\right)\right)
$$

where $1 / p_{\theta_{j}}=\left(1-\theta_{j}\right) / p_{0}+\theta_{j} / p_{1}$ for $j=0,1$. Since $\ell_{p_{\theta_{j}}}\left(2^{-n \theta_{j}}\right)$ is a parameter of the real method, the claim is established by the well known interpolation theorem for positive operators between Calderón-Lozanovsky spaces (see, e.g., [24]).

Now, we recall that if $\bar{\Phi}=\left(\Phi_{0}, \Phi_{1}\right)$ is a couple of Banach sequence spaces on $\mathbb{Z}$ which is a parameter for the method of means, then by Theorem 4.2.33 in [3], it follows that for any Banach couple $\bar{X}$,

$$
J_{\bar{\Phi}}(\bar{X})=J_{E}(\bar{X})
$$

where $E=J_{\bar{\Phi}}\left(\ell_{1}, \ell_{1}\left(2^{-n}\right)\right)$. This implies a continuous inclusion

$$
\varphi\left(X_{0}, X_{1}\right)_{p_{0}, p_{1}} \hookrightarrow J_{E}\left(X_{0}, X_{1}\right),
$$

with $E:=\varphi\left(\ell_{1}, \ell_{1}\left(2^{-n}\right)\right)_{p_{0}, p_{1}}$. In consequence, by Corollary 2.6, we obtain

$$
E \hookrightarrow \varphi\left(\ell_{p_{0}}, \ell_{p_{1}}\left(2^{-n}\right)\right)_{p_{0}, p_{1}}=\varphi\left(\ell_{p_{0}}, \ell_{p_{1}}\left(2^{-n}\right)\right) .
$$

Combining the above remarks with the well known fact (see, e.g., [8] or [3]) that the classical $K$-method as well as $J$-method determined by the same parameter of the real method generate the same interpolation spaces, we get a continuous inclusion

$$
\varphi\left(X_{0}, X_{1}\right)_{p_{0}, p_{1}} \hookrightarrow\left(X_{0}, X_{1}\right)_{\varphi\left(\ell_{p_{0}}, \ell_{p_{1}}\left(2^{-n}\right)\right)} .
$$

To end the proof we only need to show the reverse inclusion for a relatively complete Banach couple $\left(X_{0}, X_{1}\right)$ (in fact we only need to show this for the couple $\left(\ell_{\infty}, \ell_{\infty}\left(2^{-n}\right)\right)$, by the co-orbital description of the $K$-method of interpolation for this couple). Thus, assume that $\left(X_{0}, X_{1}\right)$ is relatively 
complete. Fix $x \in\left(X_{0}, X_{1}\right)_{\varphi\left(\ell_{p_{0}}, \ell_{p_{1}}\left(2^{-n}\right)\right)}$. Then apply Corollary 2.6 to obtain

$$
\xi:=\left\{K\left(2^{n}, x ; \bar{X}\right)\right\} \in \varphi\left(\ell_{p_{0}}, \ell_{p_{1}}\left(2^{-n}\right)\right)_{p_{0}, p_{1}} .
$$

Now, we invoke Proposition 2.1 to conclude that since $\left(\ell_{p_{0}}, \ell_{p_{1}}\left(2^{-n}\right)\right)$ is relatively complete, there exist positive elements $u \in \ell_{p_{0}}$ and $v \in \ell_{p_{1}}\left(2^{n}\right)$ such that

$$
K\left(t, \xi ; \ell_{p_{0}}, \ell_{p_{1}}\left(2^{-n}\right)\right) \leq K\left(t, a_{\varphi} ; \ell_{1}(u), \ell_{1}(v)\right)
$$

for all $t>0$, where $a_{\varphi}=\left\{\varphi\left(1,2^{n}\right)\right\}$.

Since

$$
K(t, x ; \bar{X}) \asymp K\left(t, \xi ; \ell_{\infty}, \ell_{\infty}\left(2^{-n}\right)\right) \leq K\left(t, \xi ; \ell_{p_{0}}, \ell_{p_{1}}\left(2^{-n}\right)\right),
$$

we obtain

$$
K(t, x ; \bar{X}) \leq K\left(t, a_{\varphi} ; \ell_{1}(u), \ell_{1}(v)\right)
$$

with $u \in \ell_{p_{0}}$ and $v \in \ell_{p_{1}}\left(2^{n}\right)$. It then follows from Proposition 2.1 that $x \in \varphi\left(X_{0}, X_{1}\right)_{p_{0}, p_{1}}$. This leads to the desired conclusion.

3. Interpolation orbits. In this section we use Banach operator ideals to study interpolation orbits. In particular, Banach operator ideal properties of inclusion maps between Banach sequence spaces allow us to get continuous inclusions between the corresponding interpolation orbit spaces and methodof-means spaces. Before we state the results, we need to introduce further notations. Let $E$ be a Banach sequence space on $\mathbb{J}$ and let $X$ be a Banach space. A vector sequence $\left\{x_{n}\right\}$ in $X$ is weakly E-summable if the scalar sequences $\left\{x^{*}\left(x_{n}\right)\right\}$ are in $E$ for every $x^{*}$ in the dual space $X^{*}$ of $X$. The space of all weakly $E$-summable sequences $\left\{x_{n}\right\}$ of a Banach space $X$ such that

$$
w_{E, X}\left(x_{n}\right):=\sup \left\{\left\|\left\{x^{*}\left(x_{n}\right)\right\}\right\|_{E} ;\left\|x^{*}\right\|_{X^{*}} \leq 1\right\}<\infty
$$

is denoted by $E^{w}(X)$.

Let $E$ and $F$ be two Banach sequence lattices on $\mathbb{J}$, and let $X$ and $Y$ be Banach spaces. An operator $T: X \rightarrow Y$ between Banach spaces is said to be $(F, E)$-summing if the induced operator $\widehat{T}$ defined on $E^{w}(X)$ by $\widehat{T}\left\{x_{n}\right\}=\left\{T x_{n}\right\}$ for $\left\{x_{n}\right\} \in E^{w}(X)$ is bounded from $E^{w}(X)$ into $F(Y)$. In this case we write

$$
\pi_{F, E}(T):=\|\widehat{T}\|_{E^{w}(X) \rightarrow F(Y)} .
$$

In what follows, we shall always assume that $E \hookrightarrow F$ since only in this case are there non-zero $(F, E)$-summing operators. We let $\Pi_{F, E}(X, Y)$ denote the space of all $(F, E)$-summing operators from $X$ to $Y$. Let us remark that under minor additional assumptions on $E$ and $F,\left(\Pi_{F, E}, \pi_{F, E}\right)$ is a Banach operator ideal in the sense of Pietsch (see [26] for details). In the case $E=\ell_{p}$ and $F=\ell_{q}$ with $1 \leq p \leq q<\infty, \Pi_{F, E}$ is the well known ideal of $(q, p)$-summing operators (see [12]). 
Recall that if $X$ and $Y$ are Banach sequence spaces on $\mathbb{J}$, the space $M(X, Y)$ of multipliers from $X$ into $Y$ is the space of all sequences $x$ such that the associated multiplication operator $X \ni y \mapsto x y$ is defined and bounded from $X$ into $Y$. The space $M(X, Y)$ equipped with the norm

$$
\|x\|_{M(X, Y)}=\sup \left\{\|x y\|_{Y} ;\|y\|_{X} \leq 1\right\}
$$

is a Banach sequence space. In the case when $Y=\ell_{1}, M\left(X, \ell_{1}\right)$ is the usual Köthe dual space $X^{\prime}$ of $X$.

The proof of the following technical result is given in [26] and is outlined below for the sake of completeness.

Lemma 3.1. Let $X$ and $Y$ be Banach spaces and let $E$ be a Banach sequence space on $\mathbb{J}$. Then every $(F, 1)$-summing operator $T: X \rightarrow Y$ is $\left(M(E, F), E^{\prime}\right)$-summing with $\pi_{M(E, F), E^{\prime}}(T) \leq \pi_{F, \ell_{1}}(T)$.

Proof. Since $\ell_{1} \hookrightarrow F$, we have $E^{\prime} \hookrightarrow M(E, F)$ for any Banach sequence lattice $E$. Let $x=\left\{x_{n}\right\} \in\left(E^{\prime}\right)^{w}(X)$. Then $\left\{\xi_{n} x_{n}\right\} \in \ell_{1}^{w}(X)$ for each $\xi=$ $\left\{\xi_{n}\right\} \in E$. This implies, by the assumption that $T: X \rightarrow Y$ is $\left(F, \ell_{1}\right)$ summing, that $\left\{\xi_{n}\left\|T x_{n}\right\|_{Y}\right\} \in F$ and

$$
\begin{aligned}
\left\|\left\{\xi_{n}\left\|T x_{n}\right\|_{Y}\right\}\right\|_{F} & \leq C \sup \left\{\sum_{n}\left|\xi_{n} x^{*}\left(x_{n}\right)\right| ;\left\|x^{*}\right\|_{X^{*}} \leq 1\right\} \\
& \leq C\|\xi\|_{E} \sup \left\{\left\|\left\{x^{*}\left(x_{n}\right)\right\}\right\|_{E^{\prime}} ;\left\|x^{*}\right\| \leq 1\right\}
\end{aligned}
$$

with $C=\pi_{F, \ell_{1}}(T)$. The above inequality implies that $\left\{T x_{n}\right\} \in M(E, F)(Y)$ and the induced map

$$
\widehat{T}:\left(E^{\prime}\right)^{w}(X) \rightarrow M(E, F)(Y)
$$

is bounded with $\|\widehat{T}\| \leq C$.

Recall that if $X$ is a Banach lattice, then the largest ideal consisting of all elements with order continuous norm is denoted by $X_{a}$. Clearly, $X_{a}=$ $\left\{x \in X ;|x| \geq x_{n} \downarrow 0\right.$ implies that $\left.\left\|x_{n}\right\| \rightarrow 0\right\}$.

Proposition 3.2. Let $\bar{E}=\left(E_{0}, E_{1}\right)$ be a couple of Banach sequence spaces on $\mathbb{J}$ and let $\xi=\left\{\xi_{n}\right\} \in\left(E_{0}+E_{1}\right)_{a}$ be such that $|\xi|>0$. If any operator $T: \bar{E} \rightarrow \bar{X}$ is such that $T: E_{j} \rightarrow X_{j}$ is $\left(F_{j}, 1\right)$-summing $(j=0,1)$ then the following continuous inclusion holds:

$$
\operatorname{Orb}(\xi, \bar{E} \rightarrow \bar{X}) \hookrightarrow J_{\Phi_{0}, \Phi_{1}}(\bar{X}),
$$

where $\Phi_{j}=M\left(E_{j}, F_{j}\right)(1 /|\xi|)$ for $j=0,1$.

Proof. Since the unit basis vectors $\left\{e_{n}\right\}$ form an unconditional basis in $\left(E_{0}+E_{1}\right)_{a}$, for any $\xi=\left\{\xi_{n}\right\} \in\left(E_{0}+E_{1}\right)_{a}$ we have

$$
\left.\xi=\sum_{n} \xi_{n} e_{n} \quad \text { (convergence in } E_{0}+E_{1}\right) \text {. }
$$


For any $T: \bar{E} \rightarrow \bar{X}$, we get

$$
\left.T \xi=\sum_{n} \xi_{n} T e_{n} \quad \text { (convergence in } X_{0}+X_{1}\right) .
$$

Further, our assumption on $T$ implies (by Lemma 3.1) that $T: E_{j} \rightarrow X_{j}$ is an $\left(M\left(E_{j}, F_{j}\right), E_{j}^{\prime}\right)$-summing operator $(j=0,1)$. Since $\left\{e_{n}\right\}$ is $F^{\prime}$-summing in each sequence Banach lattice $F$ on $\mathbb{J}$ with $w_{F^{\prime}, F}\left(e_{n}\right) \leq 1$, it follows that for $u_{n}=\xi_{n} T e_{n}$ and $j=0,1$, we have

$$
\left\|\left\{u_{n}\right\}\right\|_{\Phi_{j}\left(X_{j}\right)}=\left\|\left\{\left\|T e_{n}\right\|_{X_{j}}\right\}\right\|_{M\left(E_{j}, F_{j}\right)} \leq C \pi_{F_{j}, 1}(T) .
$$

Combining the above facts shows that $u_{n} \in X_{0} \cap X_{1}$ and $T \xi=\sum_{n} u_{n}$ (convergence in $X_{0}+X_{1}$ ), and in consequence, $x \in J_{\Phi_{0}, \Phi_{1}}(\bar{X})$.

The next result shows that in some cases we are able to describe interpolation orbits.

Proposition 3.3. Assume that $\left(E_{0}, E_{1}\right)$ and $\left(F_{0}, F_{1}\right)$ are couples of $B a-$ nach symmetric sequence spaces on $\mathbb{Z}$ such that the inclusion map $F_{j} \hookrightarrow \ell_{\infty}$ is $\left(F_{j}, 1\right)$-summing $(j=0,1)$. If $E=M\left(E_{0}, F_{0}\right)=M\left(E_{1}, F_{1}\right)$, then the following statements are true:

(i) If $\varphi \in \mathcal{P}_{0}$ and $a_{\varphi}=\left\{\varphi\left(1, t_{n}\right)\right\}$, where $\left\{t_{n}\right\}$ is the Janson sequence for $\varphi$, then

$$
\operatorname{Orb}\left(a_{\varphi},\left(E_{0}, E_{1}\left(1 / t_{n}\right)\right) \rightarrow\left(F_{0}, F_{1}\left(1 / t_{n}\right)\right)\right)=E\left(1 / \varphi\left(1, t_{n}\right)\right) .
$$

(ii) If $x \in E_{0}+E_{1}\left(2^{-n}\right)$ is such that $\varrho=K\left(\cdot, x ; E_{0}, E_{1}\left(2^{-n}\right)\right) \in \mathcal{P}^{+-}$, then

$$
\operatorname{Orb}\left(x,\left(E_{0}, E_{1}\left(2^{-n}\right)\right) \rightarrow\left(F_{0}, F_{1}\left(2^{-n}\right)\right)\right)=E\left(1 / \varrho\left(2^{n}\right)\right) .
$$

Proof. (i) Since the inclusion map $F_{1} \hookrightarrow \ell_{\infty}$ is $\left(F_{1}, 1\right)$-summing, it follows that for any weighted sequence $w$ the inclusion map $F_{1}(w) \hookrightarrow \ell_{\infty}(w)$ is also $\left(F_{1}, 1\right)$-summing. Further, $M\left(E_{1}\left(1 / t_{n}\right), F_{1}\right)=M\left(E_{1}, F_{1}\right)\left(t_{n}\right)$ shows by Proposition 3.2 (with $\xi=a_{\varphi}$ ) that

$$
\operatorname{Orb}\left(a_{\varphi},\left(E_{0}, E_{1}\left(1 / t_{n}\right)\right) \rightarrow\left(F_{0}, F_{1}\left(1 / t_{n}\right)\right)\right) \hookrightarrow \varphi\left(\ell_{\infty}, \ell_{\infty}\left(1 / t_{n}\right)\right)_{E, E} .
$$

By Theorem 7.6.2 and Lemma 7.6.3 in [29], we have

$$
\varphi\left(\ell_{\infty}, \ell_{\infty}\left(1 / t_{n}\right)\right)_{E, E}=E\left(1 / \varphi\left(1, t_{n}\right)\right) .
$$

To complete the proof, we need to show the reverse inclusion. Fix $\left\{\lambda_{n}\right\} \in$ $E\left(1 / \varphi\left(1, t_{n}\right)\right)$. Our hypothesis $E=M\left(E_{0}, F_{0}\right)=M\left(E_{1}, F_{1}\right)$ implies

$$
M\left(E_{1}\left(1 / t_{n}\right), F_{1}\left(1 / t_{n}\right)\right)=M\left(E_{1}, F_{1}\right),
$$

and hence we conclude that the diagonal map $D$ defined on $E_{0}+E_{1}\left(1 / t_{n}\right)$ by

$$
D x=\left\{\frac{\lambda_{n} x_{n}}{\varrho\left(t_{n}\right)}\right\} \quad \text { for } x=\left\{x_{n}\right\} \in E_{0}+E_{1}\left(1 / t_{n}\right)
$$


maps the couple $\left(E_{0}, E_{1}\left(1 / t_{n}\right)\right)$ continuously into $\left(F_{0}, F_{1}\left(1 / t_{n}\right)\right)$. Since $D a_{\varphi}$ $=\left\{\lambda_{n}\right\}$, the proof is complete.

(ii) First note that $\left\{2^{n}\right\}$ is the Janson sequence for $\varphi \in \mathcal{P}^{+-}$(see [17]). Further, by Theorem 4.7 in [27], it follows that $x$ and $a_{\varphi}=\left\{\varphi\left(1,2^{n}\right)\right\}$ are orbitally equivalent. This implies that the orbit spaces $\operatorname{Orb}\left(x,\left(E_{0}, E_{1}\left(2^{-n}\right)\right) \rightarrow\right.$ $\left.\left(F_{0}, F_{1}\left(2^{-n}\right)\right)\right)$ and $\operatorname{Orb}\left(a_{\varphi},\left(E_{0}, E_{1}\left(2^{-n}\right)\right) \rightarrow\left(F_{0}, F_{1}\left(2^{-n}\right)\right)\right)$ coincide up to equivalence of norms. Thus statement (i) applies.

Note that in the case when the spaces of multipliers in the above proposition do not coincide, the description of interpolation orbits is a nontrivial problem in general. In what follows, we present further results, where we do not need the assumption that the spaces of multipliers between symmetric spaces form the same space. This result can be applied to many concrete symmetric sequence spaces, e.g., Lorentz spaces $\ell_{p, q}$ and $d(w, p)$, as well as Orlicz spaces $\ell_{\varphi}$, via the results on Banach ideal properties of corresponding inclusion maps related to those spaces, presented in [9] and [10].

TheOREM 3.4. Let $\bar{X}=\left(X_{0}, X_{1}\right)$ be a couple of Banach lattices satisfying an upper 2-estimate and $x \in X_{0}+X_{1}$ be such that $\varphi(s, t)=K(s, t, x ; \bar{X})$ is non-degenerate. Assume further that $E_{j}$ is a $p_{j}$-concave Banach symmetric sequence spaces on $\mathbb{Z}, 1<p_{j} \leq 2$ for $j=0,1$. Then for any weight sequences $w_{0}$ and $w_{1}$ the following continuous inclusion holds with $F_{0}=M\left(\ell_{p_{0}}, E_{0}\right)$ and $F_{1}=M\left(\ell_{p_{1}}, E_{1}\right)$ :

$$
\operatorname{Orb}\left(x, \bar{X} \rightarrow\left(E_{0}\left(w_{0}\right), E_{1}\left(w_{1}\right)\right)\right) \hookrightarrow \varphi\left(\ell_{p_{0}}\left(w_{0}\right), \ell_{p_{1}}\left(w_{1}\right)\right)_{F_{0}, F_{1}} .
$$

Proof. Let $y=T x$ with $T: \bar{X} \rightarrow\left(E_{0}\left(w_{0}\right), E_{1}\left(w_{1}\right)\right)$. Assume that $\left\{t_{n}\right\}$ is any fundamental sequence for $\varphi$. For $x_{\varphi}:=\left\{\varphi\left(1, t_{n}\right)\right\}$, we have

$$
K(t, x ; \bar{X}) \prec K\left(t, x_{\varphi} ; \ell_{2}, \ell_{2}\left(1 / t_{n}\right)\right) .
$$

It follows by Cwikel's result (see [3, Cor. 4.4.35]) that there exists an operator $U:\left(\ell_{2}, \ell_{2}\left(1 / t_{n}\right)\right) \rightarrow\left(X_{0}, X_{1}\right)$ such that $U x_{\varphi}=x$. In consequence, we conclude that

$$
S=T U:\left(\ell_{2}, \ell_{2}\left(1 / t_{n}\right)\right) \rightarrow\left(E_{0}\left(w_{0}\right), E_{1}\left(w_{1}\right)\right)
$$

and $y=S x_{\varphi}$.

It is proved in [9] that if $E$ is a $p$-concave Banach symmetric sequence space with $1<p \leq 2$, then the inclusion map $E \hookrightarrow \ell_{p}$ is $\left(M\left(\ell_{p}, E\right), 2\right)$ summing. Thus, by the Banach ideal property, it follows that $S: \ell_{2}$ $\rightarrow \ell_{p_{0}}\left(w_{0}\right)$ is $\left(M\left(\ell_{p_{0}}, E_{0}\right), 2\right)$-summing and $S: \ell_{2}\left(1 / t_{n}\right) \rightarrow \ell_{p_{1}}\left(w_{1}\right)$ is $\left(M\left(\ell_{p_{1}}, E_{1}\right), 2\right)$-summing. Since $\left\{e_{n}\right\} \in \ell_{2}^{w}\left(\ell_{2}\right)$ and $\left.\left\{t_{n} e_{n}\right\} \in \ell_{2}^{w}\left(\ell_{2}\left(1 / t_{n}\right)\right)\right)$, it follows that

$$
\left\{\left\|S\left(e_{n}\right)\right\|_{\ell_{p_{0}}\left(w_{0}\right)}\right\} \in M\left(\ell_{p_{0}}, E_{0}\right)
$$

and

$$
\left\{t_{n}\left\|S\left(e_{n}\right)\right\|_{\ell_{p_{1}}\left(w_{1}\right)}\right\} \in M\left(\ell_{p_{1}}, E_{1}\right) .
$$


Since

$$
y=S x_{\varphi}=\sum_{n} \varphi\left(1, t_{n}\right) S\left(e_{n}\right) \quad\left(\text { convergence in } \ell_{p_{0}}\left(w_{0}\right)+\ell_{p_{1}}\left(w_{1}\right)\right),
$$

the proof is complete.

ThEOREM 3.5. Let $\bar{X}=\left(X_{0}, X_{1}\right)$ and $\bar{Y}=\left(Y_{0}, Y_{1}\right)$ be relatively complete couples of Banach lattices such that $X_{j}$ satisfies an upper $p_{j}$-estimate and $Y_{j}$ satisfies a lower $q_{j}$-estimate $(j=0,1)$. If $x \in X_{0}+X_{1}$ is such that $\varphi=K(\cdot, \cdot, x ; \bar{X}) \in \mathcal{P}_{0}$, then

$$
\operatorname{Orb}(x, \bar{X} \rightarrow \bar{Y}) \hookrightarrow \varphi\left(Y_{0}, Y_{1}\right)_{r_{0}, r_{1}},
$$

where $1 / r_{j}=\left(1 / q_{j}-1 / p_{j}\right)_{+}$for $j=0,1$.

Proof. Let $y=T x$ with $T: \bar{X} \rightarrow \bar{Y}$. Consider the sequences $x_{\varphi}=$ $\left\{\varphi\left(1, t_{n}\right)\right\}$ and $y_{\psi}=\left\{\psi\left(1, s_{n}\right)\right\}$, where $\left\{t_{n}\right\}$ and $\left\{s_{n}\right\}$ are the fundamental sequences for the functions $\varphi$ and $\psi=K(\cdot, \cdot, y ; \bar{Y}) \in \mathcal{P}_{0}$, respectively. We have

$$
K(t, x ; \bar{X}) \prec K\left(t, x_{\varphi} ; \ell_{p_{0}}, \ell_{p_{1}}\left(1 / t_{n}\right)\right)
$$

and

$$
K\left(t, y_{\psi} ; \ell_{q_{0}}, \ell_{q_{1}}\left(1 / s_{n}\right)\right) \prec K(t, y ; \bar{Y}) .
$$

It follows by Cwikel's result (see [3, Cor. 4.4.35]) that there exist operators

$$
U:\left(\ell_{p_{0}}, \ell_{p_{1}}\left(1 / t_{n}\right)\right) \rightarrow\left(X_{0}, X_{1}\right) \text { and } V:\left(Y_{0}, Y_{1}\right) \rightarrow\left(\ell_{q_{0}}, \ell_{q_{1}}\left(1 / s_{n}\right)\right)
$$

such that $U x_{\varphi}=x$ and $V y=y_{\psi}$. In consequence,

$$
S=V T U:\left(\ell_{p_{0}}, \ell_{p_{1}}\left(1 / t_{n}\right)\right) \rightarrow\left(\ell_{q_{0}}, \ell_{q_{1}}\left(1 / s_{n}\right)\right)
$$

and $S\left(x_{\varphi}\right)=y_{\psi}$. This implies, by Proposition 3.2, that

$$
y_{\psi} \in \varphi\left(\ell_{\infty}, \ell_{\infty}\left(1 / s_{n}\right)\right)_{r_{0}, r_{1}} .
$$

Now applying Corollary 2.2, we obtain $y \in \varphi\left(Y_{0}, Y_{1}\right)_{r_{0}, r_{1}}$.

Applying the result obtained in [5] (which says that if $\bar{X}=\left(X_{0}, X_{1}\right)$ is a non-trivial Banach couple then $X_{0} \cap X_{1}$ is not dense in the real method space $\left(X_{0}, X_{1}\right)_{\theta, \infty}$ for any $\left.0<\theta<1\right)$, we can easily conclude from the results obtained above that there exist a large class of couples which are not relative Calderón couples.

The following result extends a result of Ovchinnikov in [31] given for special weighted $\ell_{p}$-spaces. The proof is a modification of the one presented in [31]. We merely sketch it below in a more general case for the sake of convenience. Let us note that the key to the proof is some kind of regularization result, which uses convolutions. The idea of such constructions of regularization for sequences or functions has been used, e.g. in [32].

Proposition 3.6. Let $\left(E_{0}, E_{1}\right)$ be a couple of maximal or minimal $B a-$ nach symmetric sequence spaces on $\mathbb{Z}$ and let $w_{0}=\left\{w_{n}^{0}\right\}$ and $w_{1}=\left\{w_{n}^{1}\right\}$ be 
weighted sequences supported on $\mathbb{J}$. Assume that a positive sequence $x=\left\{\xi_{n}\right\}$ supported on $\mathbb{J}$ belongs to the Calderón-Lozanovsky space $\varphi\left(E_{0}\left(w_{0}\right), E_{1}\left(w_{1}\right)\right)$ generated by Banach sequence spaces supported on $\mathbb{J}$ and

$$
c \frac{w_{n}^{0}}{w_{n+1}^{0}} \leq \frac{\xi_{n+1}}{\xi_{n}} \leq \frac{1}{c} \frac{w_{n}^{1}}{w_{n+1}^{1}}
$$

for some $c>1$ and all $n \in \mathbb{J}$. Then there exist $\left\{\beta_{n}^{0}\right\} \in E_{0}$ and $\left\{\beta_{n}^{1}\right\} \in$ $E_{1}\left(1 / s_{n}\right)$ such that for $v_{0}=\left\{w_{n}^{0} / \beta_{n}^{0}\right\}$ and $v_{1}=\left\{w_{n}^{1} / \beta_{n}^{1}\right\}$ we have

$$
K\left(s, t, x ; \ell_{1}\left(v_{0}\right), \ell_{1}\left(v_{1}\right)\right) \prec \varphi(s, t) .
$$

Proof. By the definition of Calderón-Lozanovsky space, we have $\xi_{n}=$ $\varphi\left(\alpha_{n}^{0} w_{n}^{0}, \alpha_{n}^{1} w_{n}^{1}\right)$ with $\left\{\alpha_{n}^{0}\right\} \in E_{0}$ and $\left\{\alpha_{n}^{1}\right\} \in E_{1}\left(s_{n}\right)$. Fix $0<\varepsilon<1, j=0,1$ and define the sequence $\varepsilon^{0}=\left\{\varepsilon_{k}^{0}\right\}$ (resp., $\varepsilon^{1}=\left\{\varepsilon_{k}^{1}\right\}$ ) by $\varepsilon_{k}^{0}=(1-\varepsilon)^{-k}$ for $k \leq 0$ and $\varepsilon_{k}^{0}=0$ for $k>0$ (resp., $\varepsilon_{k}^{1}=(1-\varepsilon)^{k}$ for $0 \leq k$ and $\varepsilon_{k}^{1}=0$ for $k<0)$. Now, consider the convolutions $\beta^{j}:=\left\{\beta_{n}^{j}\right\}=\varepsilon^{j} \star \alpha^{j}, j=0,1$, defined by

$$
\beta_{n}^{0}=\sum_{k=-\infty}^{0}(1-\varepsilon)^{k} \alpha_{n-k}^{0}, \quad \beta_{n}^{1}=\sum_{k=0}^{\infty}(1-\varepsilon)^{k} \alpha_{n-k}^{1} .
$$

It is easy to see that $\alpha_{n}^{0} \leq \beta_{n}^{0}$ and $\alpha_{n}^{1} \leq \beta_{n}^{1}$ for any $n \in \mathbb{J}$. Further, it follows from $\varepsilon^{j} \in \ell_{1}$ that the map $x \mapsto \varepsilon^{j} \star x$ is bounded in the couple $\left(\ell_{1}, \ell_{\infty}\right)$. Since any maximal or minimal Banach symmetric sequence space is an interpolation space between $\ell_{1}$ and $\ell_{\infty}$, we conclude that $\beta^{0} \in E_{0}$ and $\beta^{1} \in E_{1}\left(1 / s_{n}\right)$. We show that $\left\{\beta_{n}^{0}\right\}$ and $\left\{\beta_{n}^{1}\right\}$ satisfy the required inequality. In fact, we have

$$
K\left(s, t, x ; \ell_{1}\left(v_{0}\right), \ell_{1}\left(v_{1}\right)\right)=\sum_{n \in \mathbb{J}} \xi_{n} \min \left\{\frac{s w_{n}^{0}}{\beta_{n}^{0}}, \frac{t w_{n}^{1}}{\beta_{n}^{1}}\right\} .
$$

In particular, for $s_{0}:=\beta_{k}^{0} / w_{k}^{0}$ and $t_{0}:=\beta_{k}^{1} / w_{k}^{1}$, we get

$$
K\left(s_{0}, t_{0}, x ; \ell_{1}\left(v_{0}\right), \ell_{1}\left(v_{1}\right)\right) \leq \sum_{n \leq k} \xi_{n} \frac{w_{n}^{0}}{w_{k}^{0}} \frac{\beta_{k}^{0}}{\beta_{n}^{0}}+\sum_{n>k} \xi_{n} \frac{w_{n}^{1}}{w_{k}^{1}} \frac{\beta_{k}^{1}}{\beta_{n}^{1}} .
$$

In view of our assumptions, we have

$$
\frac{\xi_{n}}{\xi_{n+1}} \frac{w_{n}^{0}}{w_{n+1}^{0}} \leq \frac{1}{c} \quad \text { and } \quad \frac{\xi_{n+1}}{\xi_{n}} \frac{w_{n+1}^{1}}{w_{n}^{1}} \leq \frac{1}{c},
$$

Combining these facts with the obvious inequalities

$$
\frac{\beta_{n+1}^{0}}{\beta_{n}^{0}} \leq \frac{1}{1-\varepsilon}, \quad \frac{\beta_{n}^{1}}{\beta_{n+1}^{1}} \leq \frac{1}{1-\varepsilon},
$$


we conclude that for $\varepsilon>0$ small enough, we get for some $q>1$,

This implies that

$$
\frac{\xi_{n}}{\xi_{n+1}} \frac{w_{n}^{0}}{w_{n+1}^{0}} \frac{\beta_{n+1}^{0}}{\beta_{n}^{0}} \leq \frac{1}{q}, \quad \frac{\xi_{n+1}}{\xi_{n}} \frac{w_{n+1}^{1}}{w_{n}^{1}} \frac{\beta_{n}^{1}}{\beta_{n+1}^{1}} \leq \frac{1}{q} .
$$

$$
\begin{array}{ll}
\frac{\xi_{n}}{\xi_{k}} \frac{w_{n}^{0}}{w_{k}^{0}} \frac{\beta_{k}^{0}}{\beta_{n}} \leq\left(\frac{1}{q}\right)^{k-n} & \text { for all } n \leq k, \\
\frac{\xi_{n}}{\xi_{k}} \frac{w_{n}^{1}}{w_{k}^{1}} \frac{\beta_{k}^{1}}{\beta_{n}^{1}} \leq\left(\frac{1}{q}\right)^{n-k} & \text { for all } n>k .
\end{array}
$$

Applying the above inequalities, we obtain

$$
\sum_{n \leq k} \xi_{n} \frac{w_{n}^{0}}{w_{k}^{0}} \frac{\beta_{k}^{0}}{\beta_{n}^{0}} \leq \frac{q}{q-1} \xi_{k}, \quad \sum_{n>k} \xi_{n} \frac{w_{n}^{1}}{w_{k}^{1}} \frac{\beta_{k}^{1}}{\beta_{n}^{1}} \leq \frac{q}{q-1} \xi_{k} .
$$

Finally, the above estimates yield

$$
\begin{aligned}
K\left(s_{0}, t_{0}, x ; \ell_{1}\left(v_{0}\right), \ell_{1}\left(v_{1}\right)\right) & \leq \frac{2 q}{q-1} \xi_{k}=\frac{2 q}{q-1} \varphi\left(\alpha_{k}^{0} w_{k}^{0}, \alpha_{1} w_{k}^{1}\right) \\
& \leq \frac{q}{q-1} \varphi\left(\beta_{k}^{0} w_{k}^{0}, \beta_{k}^{1} w_{k}^{1}\right) .
\end{aligned}
$$

In consequence, by concavity of the $K$-functional, we obtain the required estimate.

Proposition 3.7. Let $\left\{t_{n}\right\}_{n \in \mathbb{J}}$ be a fundamental sequence for $\varphi \in \mathcal{P}_{0}$ and $\left(F_{0}, F_{1}\right)$ be a couple of Banach symmetric sequence spaces on $\mathbb{Z}$. Assume that

$$
x \in \varphi\left(M\left(\ell_{p_{0}}, F_{0}\right)\left(w_{0}\right), M\left(\ell_{p_{1}}, F_{1}\right)\left(w_{1}\right)\right)
$$

and the weighted sequences $w_{0}$ and $w_{1}$ satisfy the assumptions of Proposition 3.6. Then there exists an operator $S:\left(\ell_{p_{0}}, \ell_{p_{1}}\left(1 / t_{n}\right)\right) \rightarrow\left(F_{0}\left(w_{0}\right), F_{1}\left(w_{1}\right)\right)$ such that $S\left(a_{\varphi}\right)=x$, where $a_{\varphi}=\left\{\varphi\left(1, t_{n}\right)\right\}$.

Proof. Let $E_{0}=M\left(\ell_{p_{0}}, F_{0}\right)$ and $E_{1}=M\left(\ell_{p_{1}}, F_{1}\right)$. Using Proposition 3.6, we can find $\left\{\beta_{n}^{0}\right\} \in E_{0}$ and $\left\{\beta_{n}^{1}\right\} \in E_{1}$ such that for $v_{0}:=\left\{w_{n}^{0} / \beta_{n}^{0}\right\}$ and $v_{1}:=\left\{w_{n}^{1} / \beta_{n}^{1}\right\}$, we have

$$
K\left(s, t, x ; \ell_{1}\left(v_{0}\right), \ell_{1}\left(v_{1}\right)\right) \prec \varphi(s, t) .
$$

Applying the equivalence $K\left(t, a_{\varphi} ; \ell_{p_{0}}, \ell_{p_{1}}\left(1 / t_{n}\right)\right) \asymp \varphi(1, t)$, we obtain

$$
K\left(t, x ; \ell_{p_{0}}\left(v_{0}\right), \ell_{p_{1}}\left(v_{1}\right)\right) \prec K\left(t, a_{\varphi} ; \ell_{p_{0}}, \ell_{p_{1}}\left(1 / t_{n}\right)\right) .
$$

Hence, by the Sparr theorem from [33], there exists an operator

$$
T:\left(\ell_{p_{0}}, \ell_{p_{1}}\left(1 / t_{n}\right)\right) \rightarrow\left(\ell_{p_{0}}\left(v_{0}\right), \ell_{p_{1}}\left(v_{1}\right)\right)
$$

with $T\left(a_{\varphi}\right)=x$. Since id $:\left(\ell_{p_{0}}\left(v_{0}\right), \ell_{p_{1}}\left(v_{1}\right)\right) \rightarrow\left(F_{0}\left(w_{0}\right), F_{1}\left(w_{1}\right)\right)$, we find that satisfies $S a_{\varphi}=x$.

$$
S=\operatorname{id} \circ T:\left(\ell_{p_{0}}, \ell_{p_{1}}\left(1 / t_{n}\right)\right) \rightarrow\left(F_{0}\left(w_{0}\right), F_{1}\left(w_{1}\right)\right)
$$


Theorem 3.8. Let $\bar{X}=\left(X_{0}, X_{1}\right)$ and $\bar{Y}=\left(Y_{0}, Y_{1}\right)$ be relatively complete couples of Banach lattices such that $X_{j}$ satisfies a lower $p_{j}$-estimate and $Y_{j}$ satisfies an upper $q_{j}$-estimate $(j=0,1)$. If $x \in X_{0}+X_{1}$ is such that $\varphi=K(\cdot, \cdot ; x, \bar{X}) \in \mathcal{P}_{0}$ and $\left\{t_{n}\right\}$ is the Janson sequence for $\varphi$, then

$$
\varphi\left(Y_{0}, Y_{1}\right)_{r_{0}, r_{1}} \hookrightarrow \operatorname{Orb}(x, \bar{X} \rightarrow \bar{Y}),
$$

where $1 / r_{j}=\left(1 / q_{j}-1 / p_{j}\right)+$ for $j=0,1$.

Proof. Let $y \in \varphi\left(Y_{0}, Y_{1}\right)_{r_{0}, r_{1}}$ and let $\left\{s_{n}\right\}$ be the Janson sequence for $\psi=K(\cdot, \cdot, y ; \bar{Y})$. It follows by Corollary 2.2 and Theorem 2.4 that

$$
y_{\psi}:=\left\{\psi\left(s_{n}\right)\right\} \in \varphi\left(\ell_{r_{0}}, \ell_{r_{1}}\left(1 / s_{n}\right)\right)_{r_{0}, r_{1}} \hookrightarrow \varphi\left(\ell_{r_{0}}, \ell_{r_{1}}\left(1 / s_{n}\right)\right) .
$$

Taking $w_{0}:=\{1\}$ and $w_{1}:=\left\{1 / s_{n}\right\}$, we conclude that $x:=y_{\psi}, w_{0}$ and $w_{1}$ satisfy the assumptions of Proposition 3.7. Thus, there exists an operator

$$
S:\left(\ell_{p_{0}}, \ell_{p_{1}}\left(1 / t_{n}\right)\right) \rightarrow\left(\ell_{q_{0}}, \ell_{q_{1}}\left(1 / s_{n}\right)\right)
$$

such that $S\left(x_{\varphi}\right)=y_{\psi}$, where $x_{\varphi}=\left\{\varphi\left(1, t_{n}\right)\right\}$ and $\left\{t_{n}\right\}$ is the Janson sequence for $\varphi$. Since

$$
K\left(t, x_{\varphi} ; \ell_{p_{0}}, \ell_{p_{1}}\left(1 / t_{n}\right)\right) \prec K(t, a ; \bar{X})
$$

and

$$
K(t, y ; \bar{Y}) \prec K\left(t, y_{\psi} ; \ell_{q_{0}}, \ell_{q_{1}}\left(1 / s_{n}\right)\right),
$$

applying Cwikel's result (see [3, Cor. 4.4.35]), we find that there exist operators $U:\left(X_{0}, X_{1}\right) \rightarrow\left(\ell_{p_{0}}, \ell_{p_{1}}\left(1 / t_{n}\right)\right)$ and $V:\left(\ell_{q_{0}}, \ell_{q_{1}}\left(1 / s_{n}\right)\right) \rightarrow\left(Y_{0}, Y_{1}\right)$ such that $U a=x_{\varphi}$ and $V y_{\psi}=y$. In consequence,

$$
T=V S U:\left(X_{0}, X_{1}\right) \rightarrow\left(Y_{0}, Y_{1}\right)
$$

and $T(x)=y$, i.e. $y \in \operatorname{Orb}(x, \bar{X} \rightarrow \bar{Y})$. This completes the proof.

Applying Theorems 3.5 and 3.8, we obtain a description of interpolation orbit spaces in Banach couples of weighted $L_{p}$-spaces obtained in [30] and $[31]$.

Corollary 3.9. Let $\left(L_{p_{0}}\left(u_{0}\right), L_{p_{1}}\left(u_{1}\right)\right)$ and $\left(L_{q_{0}}\left(v_{0}\right), L_{q_{0}}\left(v_{1}\right)\right)$ be $B a$ nach couples of weighted spaces. If $x \in L_{p_{0}}\left(u_{0}\right)+L_{p_{1}}\left(u_{1}\right)$ is such that $\varphi=K\left(\cdot, \cdot, x ;\left(L_{p_{0}}\left(u_{0}\right), L_{p_{1}}\left(u_{1}\right)\right)\right) \in \mathcal{P}_{0}$ and $\left\{t_{n}\right\}$ is the fundamental sequence for $\varphi$, then

$$
\operatorname{Orb}\left(x,\left(L_{p_{0}}\left(u_{0}\right), L_{p_{1}}\left(u_{1}\right)\right) \rightarrow\left(L_{q_{0}}\left(v_{0}\right), L_{q_{0}}\left(v_{1}\right)\right)\right)=\varphi\left(L_{q_{0}}\left(v_{0}\right), L_{q_{0}}\left(v_{1}\right)\right)_{r_{0}, r_{1}},
$$

with $1 / r_{j}=\left(1 / q_{j}-1 / p_{j}\right)_{+}$for $j=0,1$. In particular, if $\varphi \in \mathcal{P}^{+-}$, then

$$
\operatorname{Orb}\left(x,\left(L_{p_{0}}\left(u_{0}\right), L_{p_{1}}\left(u_{1}\right)\right) \rightarrow\left(L_{q_{0}}\left(v_{0}\right), L_{q_{0}}\left(v_{1}\right)\right)\right)=\left(L_{q_{0}}\left(v_{0}\right), L_{q_{0}}\left(v_{1}\right)\right)_{E}
$$
where $E=\varphi\left(\ell_{r_{0}}, \ell_{r_{1}}\left(2^{-n}\right)\right)$.

We remark that the results can be applied to interpolation operators. Recall that if $\bar{X}=\left(X_{0}, X_{1}\right)$ is a Banach couple and $\varphi \in \mathcal{P}$, then the abstract Marcinkiewcz space $M_{\varphi}(\bar{X})$ consists of all $x \in X_{0}+X_{1}$ such that 
$K(t, x ; \bar{X}) \prec \varphi(1, t)$, and it is equipped with the norm

$$
\|x\|=\sup \left\{\frac{K(t, x ; \bar{X})}{\varphi(1, t)} ; t>0\right\} .
$$

Note that it follows immediately by the construction of Marcinkiewicz spaces that if $T: \bar{X} \rightarrow \bar{Y}$, then $T$ maps boundedly $M_{\varphi}(\bar{X})$ into $M_{\varphi}(\bar{Y})$. The result presented below shows that under certain conditions the range space may be essentialy smaller than $M_{\varphi}(\bar{Y})$. In general, results of this type are rare and the proofs require special machinery. For related results on interpolation operators acting between abstract real method spaces, we refer to [11] and [26].

Following [3], a Banach couple $\bar{E}=\left(E_{0}, E_{1}\right)$ is called Conv $_{0}$-abundant if for any $\varphi \in \mathcal{P}_{0}$ there exists $x \in E_{0}+E_{1}$ such that $K(t, x ; \bar{E}) \asymp \varphi(1, t)$. We note that for a couple $\bar{E}$ to be $C_{0} v_{0}$-abundant, it is necessary and sufficient that there exists a non-zero element $x \in E_{0}+E_{1}$ for which $K(\cdot, x ; \bar{E}) \in \mathcal{P}^{+-}$ (see [3, Theorem 4.5.7]).

Theorem 3.10. Let $\bar{E}=\left(E_{0}, E_{1}\right)$ and $\bar{Y}=\left(Y_{0}, Y_{1}\right)$ be relatively complete couples of Banach lattices such that $E_{j}$ satisfies an upper $p_{j}$-estimate and $Y_{j}$ satisfies a lower $q_{j}$-estimate, and let $1 / r_{j}=\left(1 / q_{j}-1 / p_{j}\right)_{+}$for $j=0,1$. Assume that $\bar{E}=\left(E_{0}, E_{1}\right)$ is $\mathrm{Conv}_{0}$-abundant and $\bar{X}$ is a Banach couple such that $\bar{E}=\left(E_{0}, E_{1}\right)$ and $\bar{X}$ are relative Calderón couples. Then the following statements are true for any operator $T: \bar{X} \rightarrow \bar{Y}$ :

(i) If $\varphi \in \mathcal{P}_{0}$, then $T$ is bounded from $M_{\varphi}(\bar{X})$ into $\varphi\left(Y_{0}, Y_{1}\right)_{r_{0}, r_{1}}$.

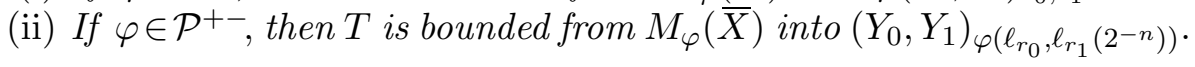

Proof. (i) Let $\varphi \in \mathcal{P}_{0}$. In view of our hypothesis, there exists $x \in E_{0}+E_{1}$ such that $K(\cdot, x ; \bar{E}) \asymp \varphi(1, \cdot)$. Since $\bar{E}$ and $\bar{X}$ are relative Calderón couples, $\operatorname{Orb}(x, \bar{E} \rightarrow \bar{X})=M_{\varphi}(\bar{X})$. To complete the proof it is enough to combine Theorem 3.5 with the obvious fact that the map $\bar{X} \mapsto \operatorname{Orb}(x, \bar{E} \rightarrow \bar{X})$ is an exact interpolation functor (see [3], [29]).

(ii) If $\varphi \in \mathcal{P}^{+-}$, then Theorem 2.7 implies

$$
\varphi\left(Y_{0}, Y_{1}\right)_{r_{0}, r_{1}}=\left(Y_{0}, Y_{1}\right)_{\varphi\left(\ell_{r_{0}}, \ell_{r_{1}}\left(2^{-n}\right)\right)},
$$

and so the proof is complete by (i).

Based on well known results on Calderón couples (see, e.g., [6], [7] and [18]), we can apply the above theorem for these couples. In particular it can be used to study interpolation of some classical operators in analysis, like singular integral operators and Hardy-Littlewood, Hilbert, Riesz, and Carleson-Hunt operators, by using well known results on boundedness of these operators between function spaces, including weighted $L_{p}$-spaces (see, e.g., [4] and [15]). We leave the details to the interested reader. 


\section{References}

[1] G. Bennett, Inclusion mappings between $l_{p}$-spaces, J. Funct. Anal. 13 (1973), 20-27.

[2] J. Bergh and J. Löfström, Interpolation Spaces. An Introduction, Springer, Berlin, 1976.

[3] Yu. A. Brudnyı̆ and N. Ya. Krugljak, Interpolation Functors and Interpolation Spaces I, North-Holland, Amsterdam, 1991.

[4] A. Cianchi, A note on two-weight inequalities for maximal functions and singular integrals, Bull. London Math. Soc. 29 (1997), 53-59.

[5] M. Cwikel and M. Mastyło, On Banach couples which satisfy the condition $\left(A_{0}, A_{1}\right)_{\theta, \infty}^{\circ}=\left(A_{0}, A_{1}\right)_{\theta, \infty}$, preprint.

[6] M. Cwikel and P. Nilsson, Interpolation of weighted Banach lattices, Mem. Amer. Math. Soc. 165 (2003), no. 787, 1-105.

[7] M. Cwikel, P. Nilsson and G. Schechtman, A characterization of relatively decomposable Banach lattices, ibid., 106-127.

[8] M. Cwikel and J. Peetre, Abstract $K$ and J spaces, J. Math. Pures Appl. 60 (1981), $1-50$.

[9] A. Defant, M. Mastyło and C. Michels, Summing inclusion maps between symmetric sequence spaces, a survey, in: Recent Progress in Functional Analysis (Valencia, 2000), North-Holland Math. Stud. 189, North-Holland, Amsterdam, 2001, 43-60.

[10] - - - - Summing inclusion maps between symmetric sequence spaces, Trans. Amer. Math. Soc. 354 (2002), 4473-4492.

[11] - , - - - Applications of summing inclusion maps to interpolation of operators, Quart. J. Math. 54 (2003), 61-72.

[12] J. Diestel, H. Jarchow and A. Tonge, Absolutely Summing Operators, Cambridge Univ. Press, 1995.

[13] V. I. Dmitriev, Duality of the interpolation methods of constants and means, Dokl. Akad. Nauk SSSR 214 (1974), 22-24 (in Russian).

[14] M. Fan, Interpolation methods of constants and means with quasi-power parameters, Math. Scand. 88 (2001), 79-95.

[15] J. García-Cuerva and J. L. Rubio de Francia, Weighted Norm Inequalities and Related Topics, North-Holland Math. Stud. 116, North-Holland, Amsterdam, 1985.

[16] J. Gustavsson and J. Peetre, Interpolation of Orlicz spaces, Studia Math. 60 (1977), $33-59$.

[17] S. Janson, Maximal and minimal methods of interpolation, J. Funct. Anal. 44 (1981), 50-73.

[18] N. J. Kalton, Calderón couples of rearrangement invariant spaces, Studia Math. 106 (1993), 233-277.

[19] S. G. Kreı̆n, Yu. I. Petunin and E. M. Semenov, Interpolation of Linear Operators, Nauka, Moscow, 1978 (in Russian); English transl.: Amer. Math. Soc., Providence, 1982.

[20] N. Ya. Krugljak, L. M. Maligranda and L. E. Persson, A Carlson type inequality with blocks and interpolation, Studia Math. 104 (1993), 161-180.

[21] J. Lindenstrauss and L. Tzafriri, Classical Banach Spaces, II, Springer, 1979.

[22] J. L. Lions et J. Peetre, Sur une classe d'espaces d'interpolation, Inst. Hautes Études Sci. Publ. Math. 19 (1964), 5-68.

[23] G. Ya. Lozanovsky, On some Banach lattices IV, Sibirsk. Mat. Zh. 14 (1973), 140155 (in Russian); English transl.: Siberian Math. J. 14 (1973), 97-108.

[24] M. Mastyło, Interpolation of linear operators in Calderón-Lozanovskii spaces, Comment. Math. Prace Mat. 26 (1986), 247-256. 
[25] M. Mastyło, Relationships between interpolation methods of means and orbits, Report No. 114/2002, Faculty of Math. \& Comp. Sci., Poznań, 19 pp..

[26] M. Mastyło and M. Milman, Interpolation of real method spaces via some ideals of operators, Studia Math. 136 (1999), 17-35.

[27] M. Mastyło and V. I. Ovchinnikov, On the relation between real and complex methods of interpolation, ibid. 125 (1997), 201-218.

[28] P. Nilsson, Interpolation of Banach lattices, ibid. 82 (1985), 135-154.

[29] V. I. Ovchinnikov, The methods of orbits in interpolation theory, Math. Rep. 1 (1984), 349-516.

[30] - On the description of interpolation orbits in couples of $L_{p}$-spaces when they are not described by the K-method, Israel Math. Conf. Proc. 5 (1992), 187-206.

[31] - Interpolation orbits in couples of $L_{p}$ spaces, C. R. Acad. Sci. Paris 334 (2002), 881-884.

[32] L. E. Persson, An exact description of Lorentz spaces, Acta Sci. Math. (Szeged) 46 (1983), 177-195.

[33] G. Sparr, Interpolation of weighted $L_{p}$-spaces, Studia Math. 62 (1978), 229-271.

Faculty of Mathematics and Computer Science

Adam Mickiewicz University

and

Institute of Mathematics

Polish Academy of Sciences (Poznań branch)

Umultowska 87

61-614 Poznań, Poland

E-mail: mastylo@math.amu.edu.pl

Received July 16, 2004

Revised version April 15, 2005 